\title{
Soft X-ray spectromicroscopy of polymers and biopolymer interfaces
}

\section{Adam P. Hitchcock}

Copyright (C) International Union of Crystallography

Author(s) of this paper may load this reprint on their own web site provided that this cover page is retained. Republication of this article or its storage in electronic databases or the like is not permitted without prior permission in writing from the IUCr. 


\section{Soft X-ray spectromicroscopy of polymers and biopolymer interfaces}

\author{
Adam P. Hitchcock \\ BIMR and Department of Chemistry, McMaster University, \\ Hamilton, ON, L8S 4M1, Canada.E-mail: aph@mcmaster.ca
}

The status of soft X-ray spectromicroscopy (near edge X-ray absorption, or NEXAFS, microscopy) is summarized, with particular emphasis on recent collaborative studies carried out by the author's group at the scanning transmission X-ray microscopy (STXM) and the photoelectron emission microscopy (PEEM) instruments at the Advanced Light Source. Results described include: characterization of phase segregation and filler particles in polyurethanes; effects of confinement on polymer self-organization; characterization of chemically structured polyurea capsule walls; and preferential interactions of proteins with chemically heterogeneous polymer surfaces. The latter is part of a systematic study of the biocompatibility of polymers used in blood contact applications.

\section{Keywords: X-ray microscopy; X-ray absorption spectroscopy;} polymers; phase segregation.

\section{Introduction}

Many natural and synthetic polymeric systems, such as engineered polymers, latexes, multilayers, biopolymers, etc., are heterogeneous. Soft X-ray spectromicroscopy (also known as near-edge X-ray absorption spectra, or NEXAFS, microscopy), in a number of variants, is finding increasing use in the analysis of these materials, on account of its ability to probe the chemical complexity of the constituents at a relevant spatial scale. This work briefly presents the main types of soft X-ray microscopy, comments on their relative strengths and weaknesses, and illustrates their performance with some examples from my current work. The versatility of the technique naturally leads to many collaborations. I recognize at the outset the essential contributions my collaborators and group members have made to the work presented here. Reviews of this field of science have been presented recently by Kirz et al. (1995), Ade (1998) and Ade \& Urquhart (2000).

In NEXAFS microscopy, inner-shell electronic excitation is used as a chemically sensitive image-contrast mechanism. NEXAFS spectra can be acquired on a small region down to the spatial resolution limit of the instrument ('microspectroscopy'), and images can be acquired at photon energies selected for their chemical sensitivity ('analytical microscopy'). In many recent applications, the full power of the combined spatial-spectral domain is exploited by automated acquisition of image sequences, which produce a three-dimensional data set which can be analyzed to extract the maximum possible chemical information from the region studied ('spectromicroscopy'). Two examples of this type of data, namely the sequence of X-ray micrographs used to map the components of a multi-component polyurethane and then to detect albumin against this polymer, have been deposited as a multimedia adjunct (.mpg file) to this paper. $\dagger$ Development of new efficient acquisition (Jacobsen et al., 2000) and data analysis procedures (Hitchcock, Koprinarov \& Kneedler, 2000;

† Supplementary data for this paper are available from the IUCr electronic archives (Reference: HI5003). Services for accessing these data are described at the back of the journal.
Koprinarov, Hitchcock, McCrory \& Childs, 2000; Osanna \& Jacobsen, $2000)$ is a very active area of microscopy.

\section{Experimental techniques}

Two main variants exist: transmission microscopy, which averages over a column of absorption and thus is generally considered 'bulk' sensitive, and electron emission microscopy, which is generally considered 'surface' sensitive. In fact, because one must use very thin samples to achieve adequate transmission of soft X-rays, the surface region contributes significantly to the transmission-mode signal. An example of a surface study by a transmission instrument is given herein. Similarly, in some electron detection microscopes, particularly PEEM instruments where the high electric field extracts low-energy electrons which have a large escape depth, the technique can be applied effectively to problems which are more of bulk than surface nature (De Stasio et al., 2000). Almost all soft X-ray transmission microscopes use zone-plate optics, either in full-field conventional transmission X-ray microscopy (TXM) or in scanning transmission Xray microscopy (STXM) configurations. The accuracy of the zone placement and the width of the outermost zone determines the spatial resolution achievable with these elements, which is about $40 \mathrm{~nm}$ in most existing instruments, although recently $25 \mathrm{~nm}$ zone plates have been reported (Denbeaux et al., 2000). Besides high spatial resolution, NEXAFS microscopy with good chemical sensitivity also requires good energy resolution. Thus a high-performance monochromated beamline is required. STXM instruments are presently only operated at undulator beamlines because of the requirement to illuminate the zone plate coherently. The brightness requirements for TXM instruments are less severe and such instruments are very successfully operated at bending-magnet sources.

Several instrument classes are available for electron emissionbased X-ray microscopy. These include point-focus devices using zone plates (scanning photoelectron microscopy, SPEM) or reflection X-ray optics [e.g. MAXIMUM, described by $\mathrm{Ng}$ et al. (1994)], and electron imaging devices using electrostatic and/or magnetic electron magnification technologies, such as PEEM. Samples for SPEM or PEEM must be ultra-high-vacuum compatible and a modicum of conductivity is required, although insulating polymers can be studied in the form of uniform thin films, with typically less than $50 \mathrm{~nm}$ of insulating layer. In contrast, TXM and STXM instruments can be readily adapted to a wide range of environments, from vacuum to atmospheric pressure, to solution studies. When traditional transmission detection is employed, TXM and STXM signals are almost completely determined by the X-ray absorption coefficient. In contrast, the signal in PEEM (and to a lesser extent SPEM) is determined not only by X-ray absorption, but also by many other factors, i.e. topography, shadowing, work function and charging. As a consequence, it has been quite straightforward to develop analysis procedures which provide quantitative chemical analysis with STXM and TXM (Loo et al., 2000), but such analysis procedures are much less developed for PEEM or SPEM.

As will be clear in the following examples, we use the NEXAFS spectral information to the maximum extent to extract quantitative chemical results, either by spectral fitting of point spectra, or by 'stack-fit' (Hitchcock, Koprinarov \& Kneedler, 2000) or singular value decomposition (SVD) (Koprinarov, Hitchcock, McCrory \& Childs, 2000) procedures applied to full image sequences or line scans. These methodologies, along with many other useful image and spectral data processing procedures are available in the IDL-based package aXis2000, which is available from the author (consult http://unicorn.mcmaster.ca/aXis2000.html). 

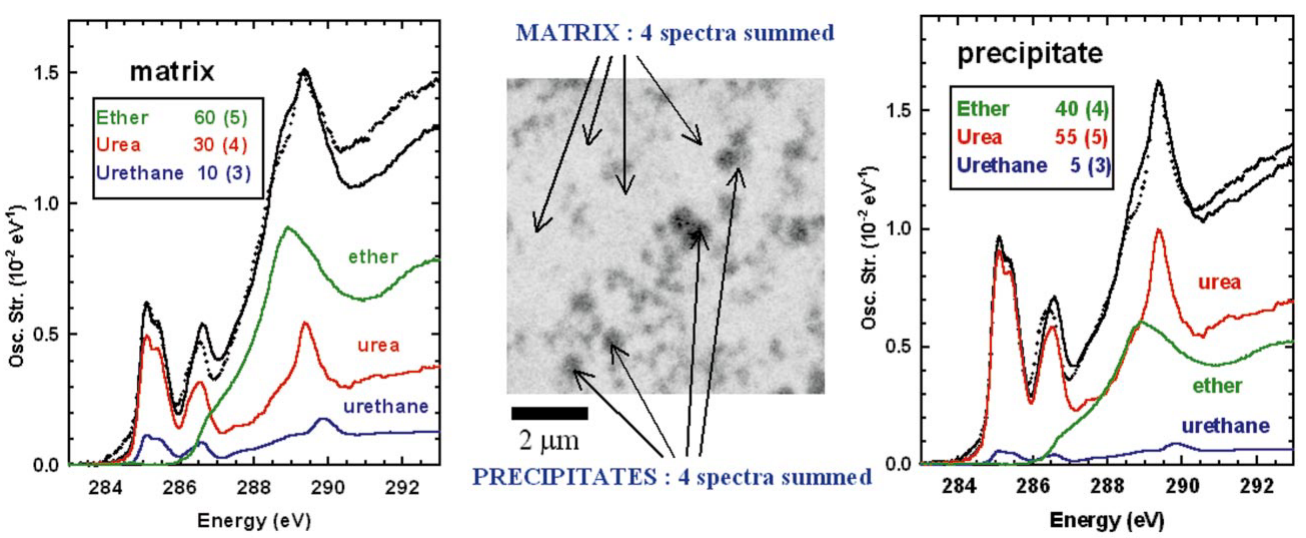

Figure 1

STXM image at $285 \mathrm{eV}$ and spot spectra of the matrix (left) and precipitate (right) regions of a high-water polyurethane foam which has macrophase precipitates. Quantitative curve-fit analysis of the matrix and precipitate spectra show that the precipitates are urea-rich. Dots are the experimental spectra [each is the sum of focused point spectra $(50 \mathrm{~nm})$ at the four indicated points], while the solid curves are the fit (thick line) and its components (thin line). (Stony Brook STXM instrument at NSLS; Rightor et al., 2000.)

\section{Examples}

\subsection{Polyurethanes}

Polyurethane polymers are formed from three major reagents: a diisocyanate (OCN-R-NCO), a polyol $\left[\mathrm{HO}-\left(R^{\prime}-\mathrm{O}\right)_{n}-R^{\prime}-\mathrm{OH}\right]$ and water, in addition to lower concentrations of chain extenders, crosslinkers, surfactants and catalysts. $R$ is typically aromatic [toluene diisocyanate (TDI) or methylenebis(phenyl isocyanate) (MDI)], while $R^{\prime}$ is an aliphatic polyether [e.g. polypropylene oxide (PPO)]. The reaction of the isocyanate and the alcohol forms a urethane linkage. The reaction of two isocyanate groups with water forms a urea linkage and $\mathrm{CO}_{2}$ gas; this gas 'blows' the foam. Polyurethane
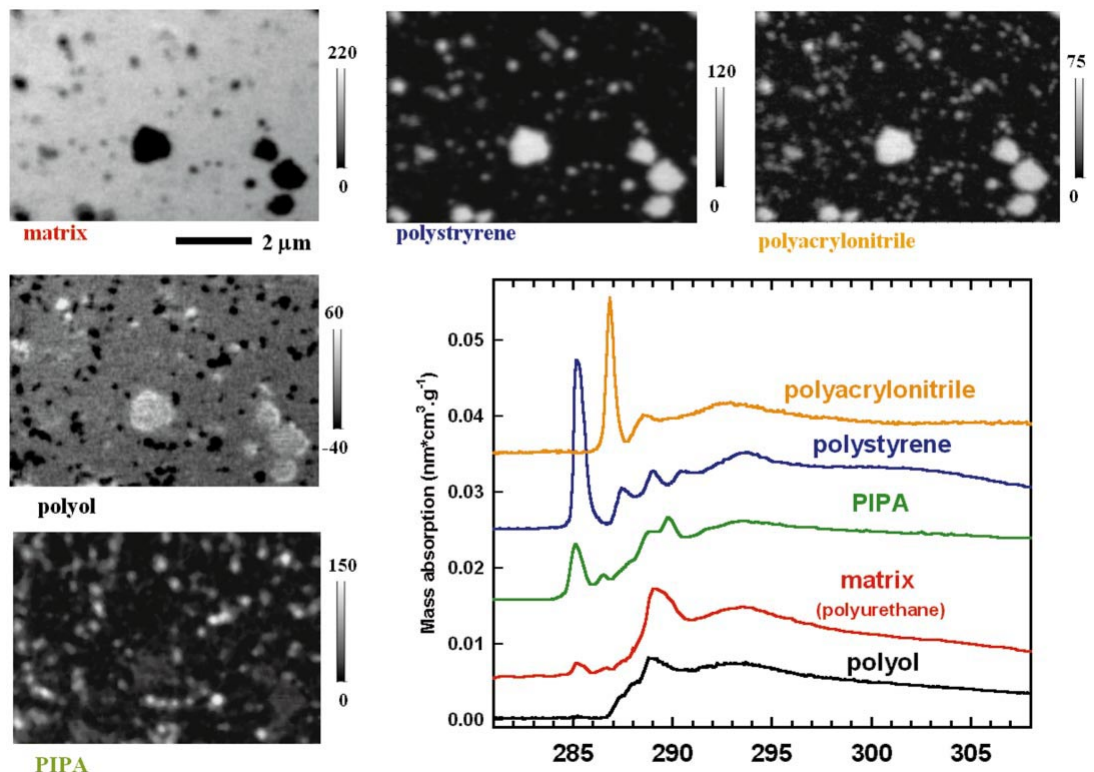

PIPA

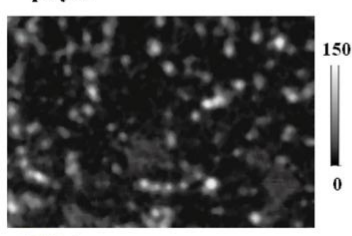

Figure 2

Maps for five components derived from stack-fit analysis of a C 1s STXM image sequence recorded on a polyurethane sample which contains PIPA and SAN (copolymer pS/pAN) filler particles. The spectral models used to derive these maps are indicated. The vertical scales of the 'relative paths' in the component maps are in $\mathrm{nm}$, assuming unit density. Note that the negative lower limit in the polyol component map is a non-physical effect of the correlation of polyol and soft-segment matrix signals. (ALS BL 7.0 STXM instrument; Hitchcock, Koprinarov, Tyliszczak et al., 2000.) foams, which are widely used in the automotive and furnishings industries, are attractive technological materials since products with widely varying physical properties (resilience, density, surface finish, etc.) can be made from essentially the same, relatively inexpensive starting materials by simple changes of formulation and fabrication procedures (Armistead \& Wilkes, 1988; Moreland et al., 1994; Herrington, 1997). Imaging and quantitative nano-scale chemical analysis of polyurethane polymers provides a means to develop rationally improved formulations for specific applications. As with many other polymer systems, polyurethanes are challenging to study since the polyether soft segment component of polyurethanes is very easily damaged, so lowdose methodologies are essential. Two examples from our polyurethane studies are provided here.

NEXAFS is readily able to distinguish urea and urethane linkages, to determine polyol content and to identify the types of $R$ and $R^{\prime}$ groups in a given polyurethane (Urquhart, Ade et al., 1999). Methods for quantitative spatially resolved mapping of the key functional groups (particularly urea and urethane) have been developed (Urquhart, Ade et al., 1999; Urquhart, Hitchcock et al., 1999). Comparison with compositions predicted from formulation chemistry indicates that NEXAFS can determine chemical content down to $\sim 5 \mathrm{~mol} \%$, with $10-20 \%$ accuracy. This is a remarkably good level of quantitation given the relatively small spectral differences which are the basis for this quantitative chemical analysis (mainly a shift of $\sim 0.4 \mathrm{eV}$ between the urea and urethane $\pi_{\mathrm{C}=\mathrm{O}}^{*}$ signals around $290 \mathrm{eV}$ ), and the extensive overlap of these key features with broad underlying $\sigma^{*}$ resonances of the dominant polyether. Good energy resolution $(\sim 0.1 \mathrm{eV})$ is essential. Fig. 1 illustrates this analysis applied to the determination of the relative urea and urethane content of matrix and precipitate regions of a macrophase segregated polyurethane (Rightor et al., 2000). As illustrated, it is possible to obtain quantitative analysis near the limits of the spatial resolution, which are $\sim 45 \mathrm{~nm}$ at the National Synchrotron Light Source (NSLS) and $\sim 90 \mathrm{~nm}$ at the Advanced Light Source (ALS) (current configuration). This analysis clearly determined that the precipitates are almost completely urea, with very little urethane. This is the first direct confirmation that precipitation in water-blown polyurethane foams is driven by water insolubility in the organic reaction mixture, which leads to urearich macrophase precipitates.

In order to make foams with higher hardness, copolymer polyols (CPPs), which are dispersions of a rigid polymer in polyether polyol, are added to the polyurethane (Gier et al., 1998). STXM is being used to help understand how CPP substances affect mechanical properties such as the elastic modulus, 
tear strength and resilience, and to aid development of improved CPP substances. While traditional chemical spectroscopies, such as infrared or NMR, are excellent at chemical speciation, they do not have adequate spatial resolution to address questions relating to the submicrometre composition of blends containing CPPs. Analytical transmission or scanning electron microscopy has superb spatial resolution, but in most cases the high-energy electron beam causes too much radiation damage and the chemical analysis capability via electron energy loss spectroscopy is inferior to modern NEXAFS microscopy on account of lower energy resolution.

Hitchcock, Koprinarov, Tyliszczak et al. (2000) have recently reported a detailed STXM study of a polyurethane with two CPP fillers: styrene-acrylonitrile (SAN) and polyisocyanate poly-additionproduct (PIPA)-based particles. Fig. 2 presents one aspect of that study: the results of a 'stack-fit' analysis (Hitchcock, Koprinarov \& Kneedler, 2000) of a C $1 \mathrm{~s}$ image sequence recorded with the ALS BL 7 STXM instrument. The spectral models, on absolute mass-absorption scales, were derived from pure materials or from NEXAFS microscopy of mixed materials, where the identity of the region investigated was known to be the species indicated. The 'images' are in fact chemical maps of each indicated component in the same area of the microtomed section, of thickness $\sim 200 \mathrm{~nm}$. Assuming unit density, the indicated vertical scales give the 'relative path' in $\mathrm{nm}$. Several interesting aspects can be seen from this work. Firstly, one can distinguish very similar chemical species. The polyol component present in the SAN particles and the polyurethane matrix are chemically very similar, except that the polyol does not have the aromatic isocyanate-derived component which is present at a level of $\sim 15 \%$ in the polyurethane matrix. Secondly, the degree of uniformity of composition of the polystyrene-polyacrylonitrile (SAN) blend can
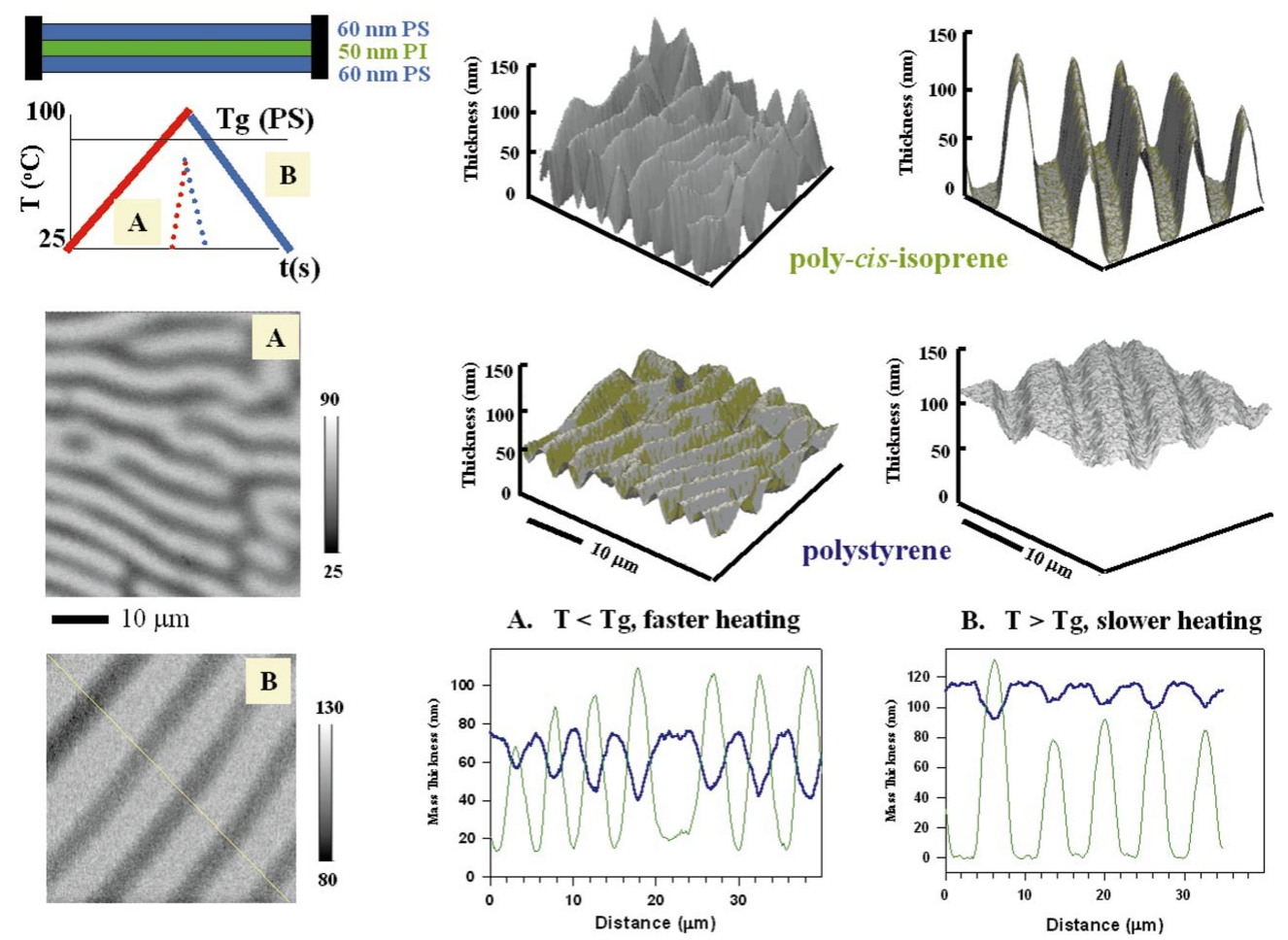

Figure 3

C 1 s STXM study of polystyrene-polyisoprene-polystyrene (PS-PI-PS) free-standing thin films. Sample $A$ was heated and cooled at $3 \mathrm{~K} \mathrm{~min}^{-1}$ to $363 \mathrm{~K}$, whereas sample $B$ was heated and cooled at $1 \mathrm{~K} \mathrm{~min}^{-1}$ to $383 \mathrm{~K}$, above the glass transition temperature $\left(T_{g}\right)$ of PS. The left-hand maps are of the PS component derived from a stack-fit analysis. Three-dimensional plots of the PS and PI component maps are presented for samples $A$ and $B$, along with line profiles across the structures. (ALS BL 7.0 STXM instrument; Koprinarov, Hitchcock, Dalnoki-Varess et al., 2000.) be readily investigated by using separate spectral components in the fit. Thirdly, there is a significant difference in the size distributions of the two classes of filler particles, as measured quantitatively by particle size analysis of component images (Hitchcock, Koprinarov, Tyliszczak et al., 2000). While this complex polymeric material can be imaged with better spatial resolution using TEM, the richness and precision of chemical information provided by NEXAFS spectromicroscopy far exceed anything that could be obtained by analytical TEM, even with staining techniques. In addition to providing a dramatic demonstration of the power of STXM microanalysis of chemical microstructure of filler-particle-reinforced polyurethanes to improve the foam properties.

\subsection{Self-organization of confined free-standing polymer films}

Patterning of thin polymer films is attracting increasing interest, both from a fundamental point of view and on account of potential technological applications. However, the thermal stability of these films can be a limitation on account of hole formation driven by possibility of control over this instability, which in turn raises possibilities for unique self-assemblies of polymers on surfaces patterned submicrometre length scales.

In order to separate thin film-thin film interactions from thin filmsubstrate interactions, studies are being performed by the Dutcher group (Dalnoki-Varess et al., 1999) on free-standing trilayer structures, consisting of a thin layer of a low-melting rubbery polymer (polyisoprene, PI) between two thin layers of a higher-melting rigid polymer (polystyrene, PS). Optical microscopy readily reveals fascinating patterns that form spontaneously or after gentle annealing of PS-PI-PS trilayers. A mechanism of formation involving long-range dispersion forces has been proposed (Dalnoki-Varess et al., 1999). However, the optical studies could not determine to what extent each layer contributes to the patterning through its spatial reorganization. Such information is required to evaluate the proposed model quantitatively. Quantitative mapping with STXM readily distinguishes the patterning in the PS layers from that in the PI layer. Fig. 3 illustrates quantitative composition maps for PS and PI derived from C $1 \mathrm{~s} \mathrm{STXM}$ of two different PS-PIPS trilayer films (Koprinarov, Hitchcock, Dalnoki-Varess et al., 2000). As expected, the softer polyisoprene provides most of the patterning. However, significant patterning, which is out-of-phase with the PI pattern, is also measured in the polystyrene 
layers. Surprisingly, the degree of this patterning was found to be larger in a sample annealed to a lower temperature (but more rapidly) than in a sample annealed more slowly to a higher temperature. This counter-intuitive result suggests that kinetics may be an important factor in controlling the pattern formation.

\subsection{Microstructure of polyurea capsule walls}

Polymers are frequently used to encapsulate other chemicals for a variety of purposes, e.g. packaging, transport, controlled release of drugs, nutrients, or pesticides, etc. We have used STXM to detect and quantify the chemical structure of walls, of sub-micrometre thickness, of polyurea capsules (Croll et al., 2000). The capsules are produced by interfacial polymerization: reaction between amine and isocyanate at the interface between organic and aqueous phases in a dispersion of a 1:10 mixture of MDI and xylene in an aqueous solution of ethylene diamine. In addition to the amine-isocyanate reaction which produces an asymmetric aromatic aliphatic urea, there is a competing reaction between the MDI and water which produces a symmetric diaromatic urea. The temporal and spatial aspects of this competition can lead to the formation of gradients in the chemical structure of the walls of these capsules. These are postulated to occur as a result of (i) pre-reaction with water in an amine-free emulsification step, which results in an outer skin of symmetric urea; (ii) preferential diffusion and reaction of amine relative to water during the reaction, which leads to a central region rich in asymmetric urea; and (iii) postsynthesis curing when capsules are stored in water, in which water enters the capsule and reacts with residual isocyanate to form innerwall structures rich in symmetric urea.

Fig. 4 shows images, chemical compositional maps and profiles of the structure of the capsule walls from two different polyurea capsule formulations. In one, a very short emulsification period was used and the outer skin of symmetric urea is relatively thin. In the other, emulsification was carried out for an extended $2 \mathrm{~h}$ period and a thicker outer skin of symmetric urea is found. The distributions of asymmetric (amine reaction) and symmetric (water reaction) ureas across these two membranes derived from C $1 \mathrm{~s}$ STXM image sequences are qualitatively consistent with the model of competitive reactions. Analysis of diffusion and reaction kinetics is now in progress to model quantitatively the capsule-wall formation process. The ability to fabricate structured walls with controlled chemical gradients could allow significant improvements in capsule properties for specific applications.

\subsection{Protein-polymer interface studies}

Increasingly, medicine uses artificial devices to replace or reinforce diseased body parts or to replace defective body functions. One
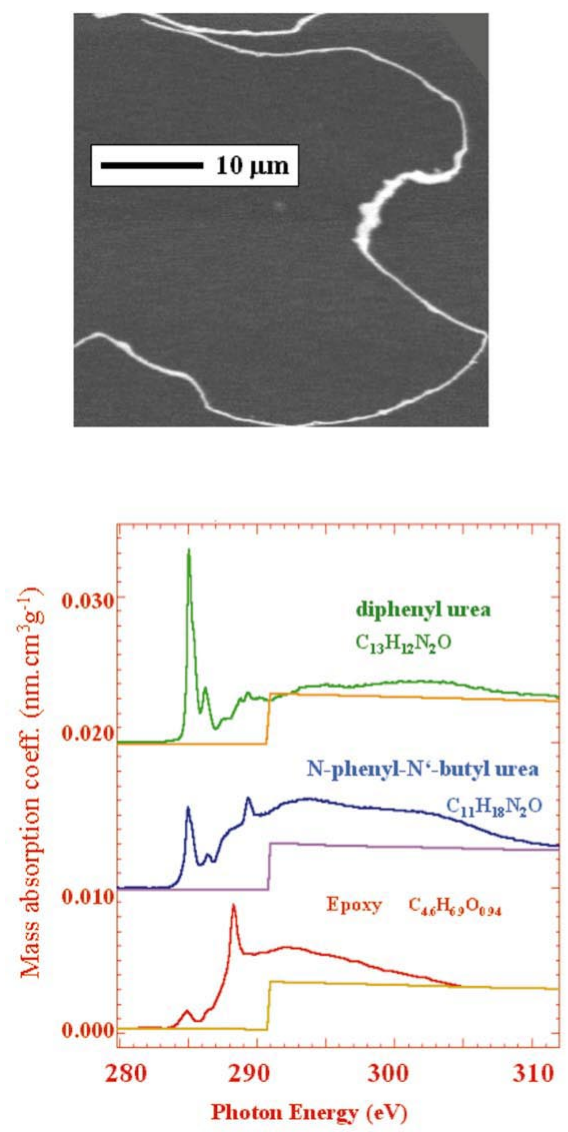
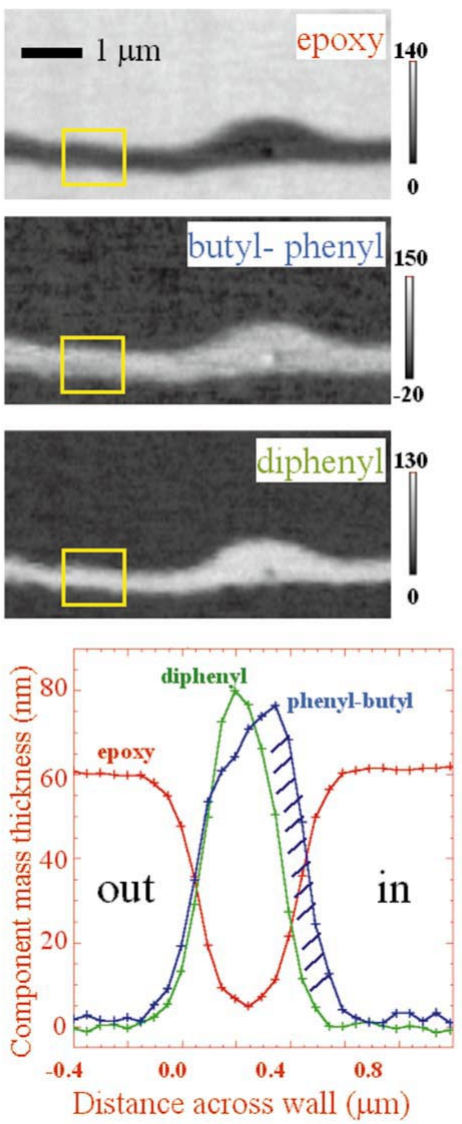
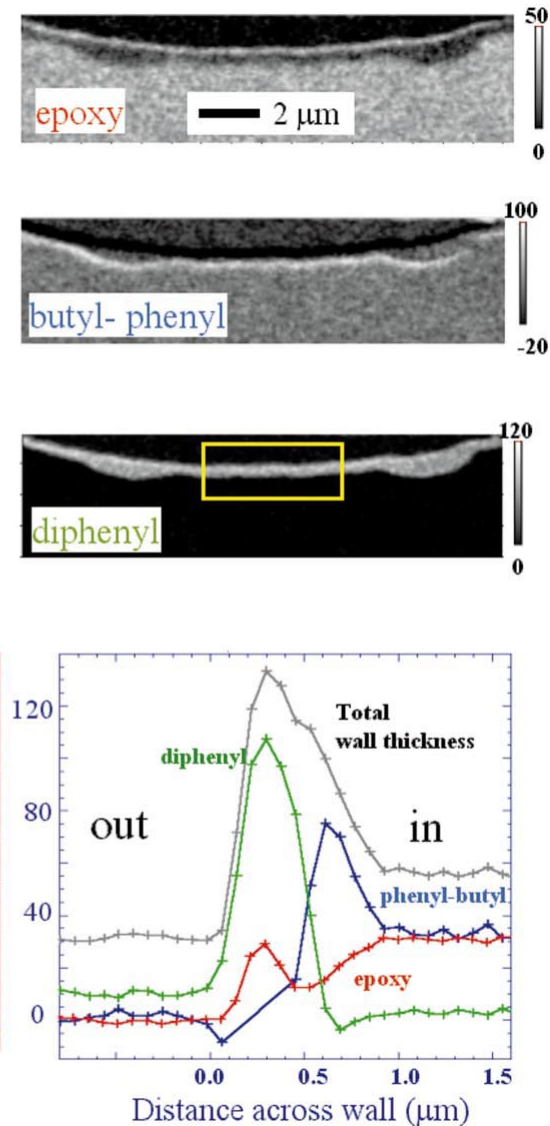

Figure 4

C $1 \mathrm{~s}$ STXM study of polyurea capsules. The upper left shows an image at $285 \mathrm{eV}$ of a microtomed section containing a nearly complete capsule embedded in epoxy. The lower left shows the C $1 \mathrm{~s}$ spectra of the epoxy, butylphenylurea (asymmetric model) and diphenylurea (symmetric model) on a mass absorption scale set by matching to the elemental response given by summing mass absorption coefficient spectra (Henke et al., 1993). The spectra of small molecules with similar local structure have been used to model the latter two components. The center panel displays derived component maps and average line profiles of each component across the capsule wall in the indicated region. The right panel shows the corresponding signals for a different capsule wall: one prepared with a longer emulsification step, which leads to a thick outer skin of symmetric urea. Note that different distance scales are used for the two different capsule samples. (ALS BL 7.0 STXM instrument; Croll et al., 2000.) 
example is the use of devices made from synthetic polymers in hemodialysis. Activation of blood coagulation, thrombosis and the immune system are key problems here and in the many other situations where blood is in contact with artificial surfaces (heart-lung bypass for open-heart surgery, artificial heart valves, heart-assist devices, arterial grafts, intravascular stents, all involving thousands of patients daily worldwide). These effects are known to be initiated by interactions of blood proteins with the surface of the material, which is often a polymer. We are using NEXAFS microscopy to assist the group of John Brash (Chemical Engineering, McMaster) in efforts to develop polymer surfaces which prevent or minimize these phenomena. This motivates our thrust to develop effective X-ray spectromicroscopy tools to investigate protein-polymer interactions, ultimately at the solid-solution interface, but as an interim, in vacuum with PEEM, or in He atmosphere (STXM). There are significant challenges involved in this work, since the obvious surface analytical approach, electron yield detection, is often difficult or impossible with polymers on account of charging. For the past year we have been exploring the detection limits of both STXM and PEEM for a variety of proteins on a variety of polymer substrates. The polyurethane materials currently preferred for blood-contact applications have a microphase segregation that is on a sub-ten-micrometre scale, finer by a factor of 5-10 than the spatial resolution of existing soft X-ray spectromicroscopes. Thus we have been using a range of alternate materials: polyurethanes with macrophase segregation (as in Fig. 1) or with embedded filler particles (as in Fig. 2), as well as polystyrene-polymethylmethacrylate (pS-pMMA) blends. Our results to date indicate that even though STXM is essentially a 'bulk' technique, monolayer detection is possible although at the current sensitivity limits of this technique. In particular, the sensitivity to an adsorbed layer depends on the spectrum of the underlying polymer, since the most reliable detection scheme relies on use of the full spectral signature through
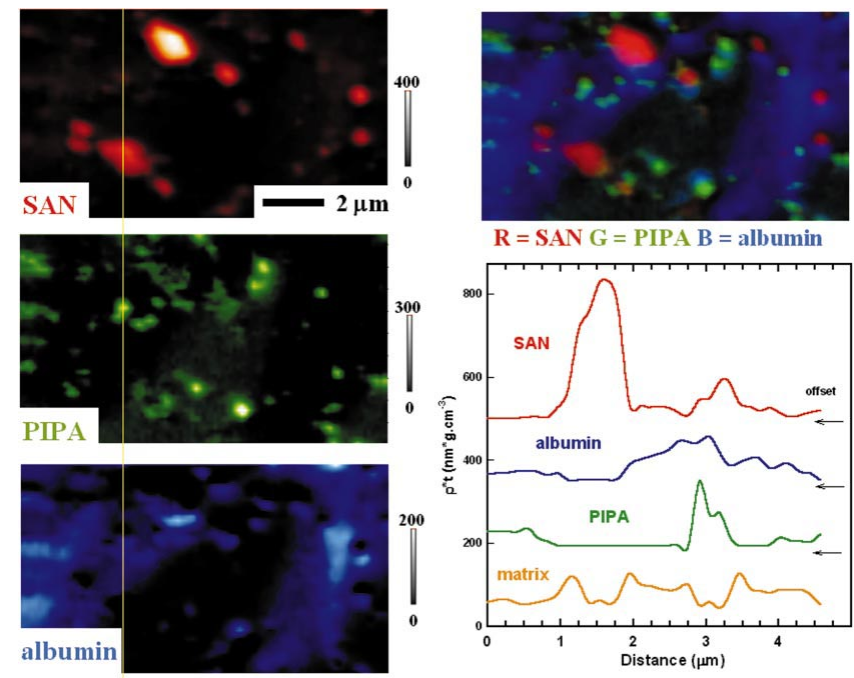

albumin

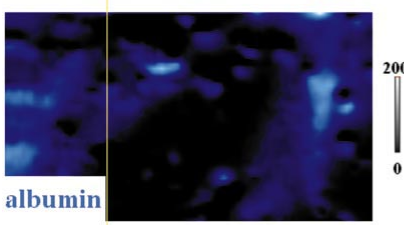

\section{Figure 5}

Component maps derived from a C $1 \mathrm{~s}$ STXM image sequence of human serum albumin adsorbed on a polyurethane with PIPA and SAN filler particles (see Fig. 2 for further chemical microanalysis of the polymer substrate). The lower right-hand plot gives the mass thickness of each of the indicated components along the line indicated on the composition images. The color composite image (upper right) is a byte-scaled combination of the SAN (red), PIPA (green) and protein (blue) components, in order to display the spatial relationship of the protein relative to the two types of filler particles. (ALS BL 7.0 STXM instrument.) image sequence analysis, rather than simply imaging at one specific wavelength.

Fig. 5 shows results of this approach for albumin adsorbed on a microtomed section of the two-filler polyurethane system presented in Fig. 2. The microtomed surface is believed to have the three classes of chemical constituents, namely polyether-rich matrix, SAN and PIPA, exposed at the surface. The protein was introduced by a true adsorption process from a very dilute buffered solution $\left(0.1 \mathrm{mg} \mathrm{ml}^{-1}\right)$ with thorough rinsing. The amount detected is in the monolayer range in some regions (each albumin molecule is $3 \times 8 \mathrm{~nm}$ ) and there is clear indication of adsorption preference in the order PIPA > polyurethane matrix (polyether) $>$ SAN (styrene-acrylonitrile). The sequence of STXM images that was used to derive the component maps presented in Fig. 5 is available as supplementary material (.mpg movie).

It is interesting to contrast the current capabilities of STXM and PEEM for studies of protein adsorption on polymers. Fig. 6 presents results of a PEEM study of albumin adsorption on a polytetramethylene oxide-ethylenediamine-MDI (PTMO-ED-MDI) polyurethane, used in blood-contact applications. The polymer was solvent cast to form a thin film on a silicon wafer coated with native oxide. Unfortunately, the resulting film was rather uneven, which generates large topographical contrast and probably means that the adsorption of the protein is driven by mechanical as well as chemical binding. Even so, the N 1 s NEXAFS readily distinguishes the loca-
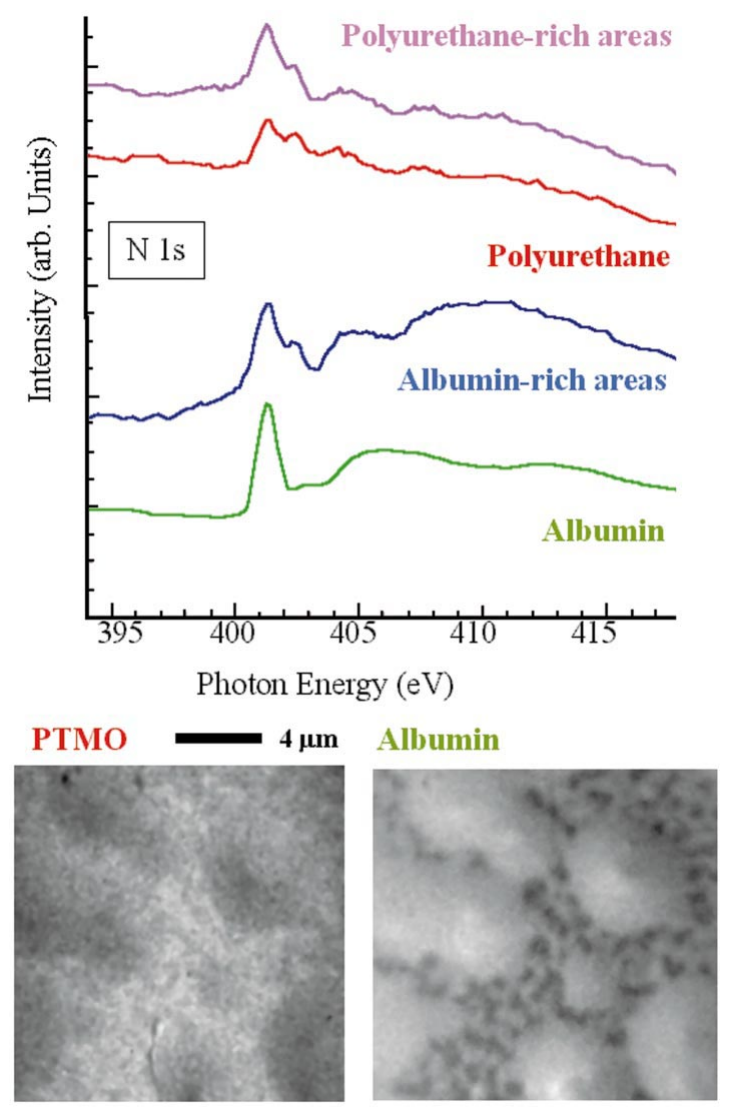

Figure 6

Component maps derived from N $1 \mathrm{~s}$ PEEM image sequence of albumin adsorbed onto the surface of a polyurethane (PTMO-ED-MDI) solvent cast onto a silicon wafer. The plot compares spectra of the pure materials with spectra extracted from the region with highest signal strength of each component. (ALS BL 7.3 PEEM instrument.) 
tion of the albumin on the polyurethane surface. The distribution of the protein appears to reflect avoidance of thicker regions of the polyurethane cast film. This could be caused simply by mechanical attachment in hollows on a rough polymer film, or it could reflect a preference of albumin to bind to the oxide surface of the bare silicon wafer. One of the limitations of the PEEM technique is that it is very difficult to quantify signal intensities since there are many factors other than the X-ray absorption coefficient that determine the sample response.

\section{Future directions}

NEXAFS spectromicroscopy is clearly a powerful analytical tool for chemical studies of sub-micrometre structure. Scanning zone-plate microscopy has special advantages for radiation-sensitive materials and for studies in the wet state. Significant improvements are just emerging or are expected in the coming few years. These include: cryomicroscopy, which is now available at several TXM facilities (ALS, BESSY) and at the NSLS STXM instrument (Maser et al., 2000; Wang et al., 2000); higher spatial resolution with improved zone plates (Denbeaux et al., 2000); phase-contrast (Miao et al., 1998) and dark-field (Chapman et al., 1996) detection techniques; in situ studies of mechanically, thermally and chemically perturbed materials; electron-yield surface detection in vacuum-operated STXM (Hitchcock et al., 1998); luminescence and X-ray fluorescence detection; new PEEM instruments with projected resolution below $5 \mathrm{~nm}$.

In terms of science, continued improved performance will allow more detailed studies of blends, copolymers, self-assembly and nanopatterned polymer structures. It is widely expected that a new level of detail of polymer organization will be measurable when orientationcontrast studies (Smith \& Ade, 1996) become much more routine with the introduction, in a few years, of STXM on beamlines attached to elliptically polarized undulators that can produce linearly polarized light with an arbitrary orientation.

Finally, a large portion of the polymer and biomaterial studies carried out to date have been performed by, or in collaboration with industrial researchers. The rapid recognition by industry of the remarkable value of this technique attests further to the added value NEXAFS microscopy provides to practical problem solving relative to other, more accessible, laboratory-based analytical microscopy techniques. The future of NEXAFS microscopy is bright!

This work is funded by the Natural Science and Engineering Research Council (Canada). The ALS STXM instrument was developed by T. Warwick (ALS), B. Tonner (UWM) and collaborators, with support from the US DOE under contract DE-AC0376SF00098. Zone plates used at the ALS were provided by Eric Anderson of CXRO, LBNL. I thank ALS staff for much assistance and expert operation. The Stony Brook STXM instrument was developed by the groups of J. Kirz and C. Jacobsen, with support from the Office of Biological and Environmental Research, US DOE under contract DE-FG02-89ER60858, and the NSF under grant DBI9605045. The zone plates were developed by S. Spector and C. Jacobsen of Stony Brook and Don Tennant of Lucent Technologies Bell Labs, with support from the NSF under grant ECS-9510499. This work is the result of extensive collaborations with many others. I wish to thank especially Tolek Tyliszczak, Harald Stöver, John Brash, Rena Cornelius, Yew-Meng Heng, Ivo Koprinarov and Cynthia
Morin (McMaster University); John Dutcher, K. Dalnoki-Varess and C. Murray (University of Guelph); Harald Ade and Archie Smith (North Carolina State University); Stephen Urquhart (U. Saskatchewan), Ed. Rightor, Werner Lidy, Fred Hayes, Mike Dineen and Gary E. Mitchell (Dow Chemical); and Rick Steele, George Meigs and Tony Warwick (Advanced Light Source, Berkeley).

\section{References}

Ade, H. (1998). Experimental Methods in the Physical Sciences, Vol. 32, edited by J. A. R. Samson \& D. L. Ederer, pp. 225-261. New York: Academic Press.

Ade, H. \& Urquhart, S. G. (2000). In Chemical Applications of Synchrotron Radiation, edited by T. K. Sham. Singapore: World Scientific. In the press. Armistead, J. P. \& Wilkes, G. L. (1988). J. Appl. Polym. Sci. 35, 601-629.

Chapman, H., Jacobsen, C. \& Williams, S. (1996). Ultramicroscopy, 62, $191-$ 213.

Croll, L. M., Koprinarov, I., Hitchcock, A. P., Li, W. H. \& Stöver, H. (2000). In preparation.

Dalnoki-Varess, K., Nickel, B. G. \& Dutcher, J. R. (1999). Phys. Rev. Lett. 82, 1486.

Denbeaux, G., Anderson, E., Chao, W., Eimueller, T., Fischer, P., Johnson, L. Koehler, M., Le Gros, M., Lucero, A., Olynick, D. \& Attwood, D. (2000). J. Electron Spectrosc. In the press.

De Stasio, G., Gilbert, B., Frazer, B., Mercanti, D., Casalbore, P., Pllini, R., Larocca, L. M., Nealson, K., Conrad, P. G., Douglas, S., Belz, A. P., Labrenz, M. \& Banfield, J. F. (2000). J. Electron Spectrosc. In the press.

Gier, D. R., O'Neill, R. E., Adams, M. R., Priester, R. D Jr, Lidy, W. A., Rightor, E. G., Barnes, C. G. \& Davis, B. L. (1998). Filler, Hard Phases and Copolymer Polys - Their Different Mechanism to Reinforce Flexible Polyurethane Foams, pp. 278-299. Dallas: Society of Plastics Institute.

Henke, B. L., Gullikson, E. M. \& Davis, J. C. (1993). At. Nucl. Data Tab. 54, 181. See also http://www-cxro.lbl.gov/optical_constants/.

Herrington, R. (1997). Flexible Polyurethane Foams, 2nd ed. The Dow Chemical Company, Freeport, TX 77541, USA.

Hitchcock, A. P., Koprinarov \& Kneedler, E. M. (2000). In preparation.

Hitchcock, A. P., Koprinarov, I., Tyliszczak, T., Rightor, E. G., Mitchell, G. E., Dineen, M. T., Hayes, F., Lidy, W., Priester, R. D., Urquhart, S. G., Smith, A. P. \& Ade, H. (2000). Ultramicroscopy. In the press.

Hitchcock, A. P., Tyliszczak, T., Urquhart, S. G., Ade, H. W., Murti, K., Gerroir, P., Rightor, E. G., Lidy, W., Dineen, M. T., Mitchell, G. E., Steele, W. S., Meigs, G. \& Warwick, T. (1998). ALS Compendium, LBNL publication (http://alspubs.lbl.gov/compendium/).

Jacobsen, C., Wirick, S., Flynn, G. \& Zimba, C. (2000). J. Microsc. 197, 173-179.

Kirz, J., Jacobsen, C. \& Howells, M. (1995). Q. Rev. Biophys. 28, 33.

Koprinarov, I., Hitchcock, A. P., Dalnoki-Varess, K., Murray, C., Dutcher, J. \& Ade, H. (2000). In preparation.

Koprinarov, I., Hitchcock, A. P., McCrory, C. T. \& Childs, R. F. (2000). J. Phys. Chem. B. To be submitted.

Loo, B. W. Jr, Sauerwald, I. M., Hitchcock, A. P. \& Rothman, S. S. (2000). J. Microsc. In the press.

Maser, J., Osanna, A., Wang, Y., Jacobsen, C., Kirz, J., Spector, S., Winn, B. \& Tennant, D. (2000). J. Microsc. 197, 68-79.

Miao, J., Sayre, D. \& Chapman, H. N. (1998). J. Opt. Soc. Am. A, 15, $1662-$ 1669.

Moreland, J. C., Wilkes, G. L., Turner, R. B. \& Rightor, E. G. (1994). J. Appl. Polym. Sci. 52, 1459-1476.

Ng, W., Ray-Chaudhuri, A. K. \& Liang, S. (1994). Nucl. Instrum. Methods Phys. Res. A, 327, 422-430.

Osanna, A. \& Jacobsen, C. (2000). AIP Conf. Proc. 507, 350-355.

Rightor, E. G., Mitchell, G. E., Urquhart, S. G., Smith, A. P., Ade, H. \& Hitchcock, A. P. (2000). In preparation.

Smith, A. P. \& Ade, H. (1996). Appl. Phys. Lett. 69, 3833-3836.

Urquhart, S. G., Ade, H., Smith, A. P., Hitchcock, A. P., Rightor, E. G. \& Lidy, W. (1999). J. Phys. Chem. B, 103, 4603-4610.

Urquhart, S. G., Hitchcock, A. P., Smith, A. P., Ade, H., Lidy, W., Rightor, E. G. \& Mitchell, G. E. (1999). J. Electron Spectrosc. 100, 119-135.

Wang,Y., Jacobsen, C., Maser, J. \& Osanna, A. (2000). J. Microsc. 197, 80-93. 